\title{
Free energies of stalk formation in the
}

\section{lipidomics era}

\author{
Chetan S. Poojari, Katharina C. Scherer, and Jochen S. Hub* \\ Theoretical Physics and Center for Biophysics, Saarland University, Saarbrücken, Germany \\ E-mail: jochen.hub@uni-saarland.de \\ Phone: +49 (0)681 302-2740. Fax: +49 (0)681 302-2748
}

\begin{abstract}
Many biological membranes are asymmetric and exhibit complex lipid composition, comprising hundreds of distinct chemical species. Identifying the biological function and advantage of this complexity is a central goal of membrane biology. Here, we study how membrane complexity controls the energetics of the first steps of membrane fusions, that is, the formation of a stalk. We first present a computationally efficient method for simulating thermodynamically reversible pathways of stalk formation at near-atomic resolution. The new method reveals that the inner leaflet of a typical plasma membrane is far more fusogenic than the outer leaflet, which is likely an adaptation to evolutionary pressure. To rationalize these findings by the distinct lipid compositions, we computed $\sim 200$ free energies of stalk formation in membranes with different lipid head groups, tail lengths, tail unsaturations, and sterol content. In summary, the simulations reveal a drastic influence of the lipid composition on stalk formation and a comprehensive fusogenicity map of many biologically relevant lipid classes.
\end{abstract}




\section{Introduction}

Eukaryotic cellular membranes contain more than ten lipid classes, while each class comprises hundreds of different chemical species. ${ }^{1,2}$ The complexity of membranes is further increased by the membrane asymmetry, that is, by distinct lipid compositions in the two leaflets. In the plasma membrane of mammals, sphingolipids are typically enriched in the outer leaflet of the plasma membrane, whereas phosphatidylethanolamine (PE) and phosphatidylserine (PS) are enriched in the inner leaflet. ${ }^{3}$ Apart from different head groups, lipid species differ by the length and unsaturation of the fatty acid tails. Recent lipidomics studies showed that polyunsaturated lipids are strongly enriched in the inner leaflet. ${ }^{4}$ Understanding why biological cells synthesize and maintain this complex lipid repertoire, that is, defining the biological function and advantage of specific lipid compositions, remains a central goal of membrane biophysics.

Here, we study how complex lipid compositions control the early stages of membrane fusion. Fusion is critical for many processes that involve the transport of cargos across membranes such as exocytosis, neurotransmission, infection of cells by enveloped viruses, fertilization, and intracellular transport. ${ }^{5-8}$ Membrane fusion occurs with the help of fusion proteins because fusion requires the two opposing lipid membranes to overcome a large dehydration barrier. The process starts with two membranes in the lamellar phase at the equilibrium distance of $2-3 \mathrm{~nm}$, followed by bridging of two opposing membranes by lipid acyl chains to establish a point-like protrusion. The lipids of the two contacting, proximal leaflets mix to form the initial transient hemifusion stalk. The stalk expands to allow the formation and expansion of a fusion pore. ${ }^{9-11}$

Because intermediate structures along the fusion pathway involve highly curved membranes, the intrinsic curvature of lipids influences the kinetics of fusion. For instance, when added to the proximal leaflets, inverted cone-shaped lysophosphatidylcholine (LPC) inhibits stalk formation because it is incompatible with the large negative curvature at the stalk rim. Inversely, cone-shaped unsaturated PE lipids or diacylglycerol promote stalk forma- 
tion. ${ }^{10,12,13}$ Here, the unsaturated fatty acids render the lipids more cone-shaped because the double bonds increase the tail disorder and, hence, the effective volume of the lipid tails. ${ }^{13}$ However, in addition to such effect by the geometric lipid shape, the increased abundance of double bonds increases the conformational flexibility of the lipids, thereby allowing the membrane to adapt more easily to curvature, ${ }^{14}$ which may further promote fusion.

Theoretical and computational approaches have established possible fusion pathways and the underlying free energy landscapes, however, only few studies focused on the role of the lipid composition during fusion. Early studies employed continuum descriptions, which model the role of lipids in terms of the effective spontaneous curvature and membrane rigidity, ${ }^{15}$ as well as minimal coarse-grained lipids models in conjunction with Monte-Carlo, fieldtheoretic, or Brownian dynamics methods. ${ }^{16-18}$ Complementary, to account for the chemical specificity of lipid-lipid interactions, fusion was studied with atomistic and coarse-grained (CG) molecular dynamics (MD) simulations, ${ }^{19-21}$ discussed in several excellent reviews. ${ }^{22-24}$ PE lipids were found to enhance fusion during simulations as expected from their negative intrinsic curvature, ${ }^{25-27}$ however the effects of other head groups, sterols, or degrees of unsaturation have hardly been considered despite their abundance in biological membranes.

Computationally efficient free energy calculations of fusion require the definition of one or several reaction coordinates (or order parameters) along the fusion pathway; however, finding good coordinates for complex transitions is non-trivial. With poor coordinates, hysteresis problems emerge, barriers may be integrated out, and simulations proceed along non-reversible pathways. To avoid such problems, a recent elegant MD study used the string method to optimize the minimum free energy pathway of stalk formation, parametrized by the three-dimensional density of the apolar lipid tail beads. ${ }^{27}$ However, because the string method is computationally demanding, it becomes prohibitive for high-throughput studies. In this study, we introduce a reaction coordinate for stalk formation that allows computationally highly efficient and thermodynamically reversible free energy calculations of stalk formation using common umbrella sampling simulations. The potentials of mean force 

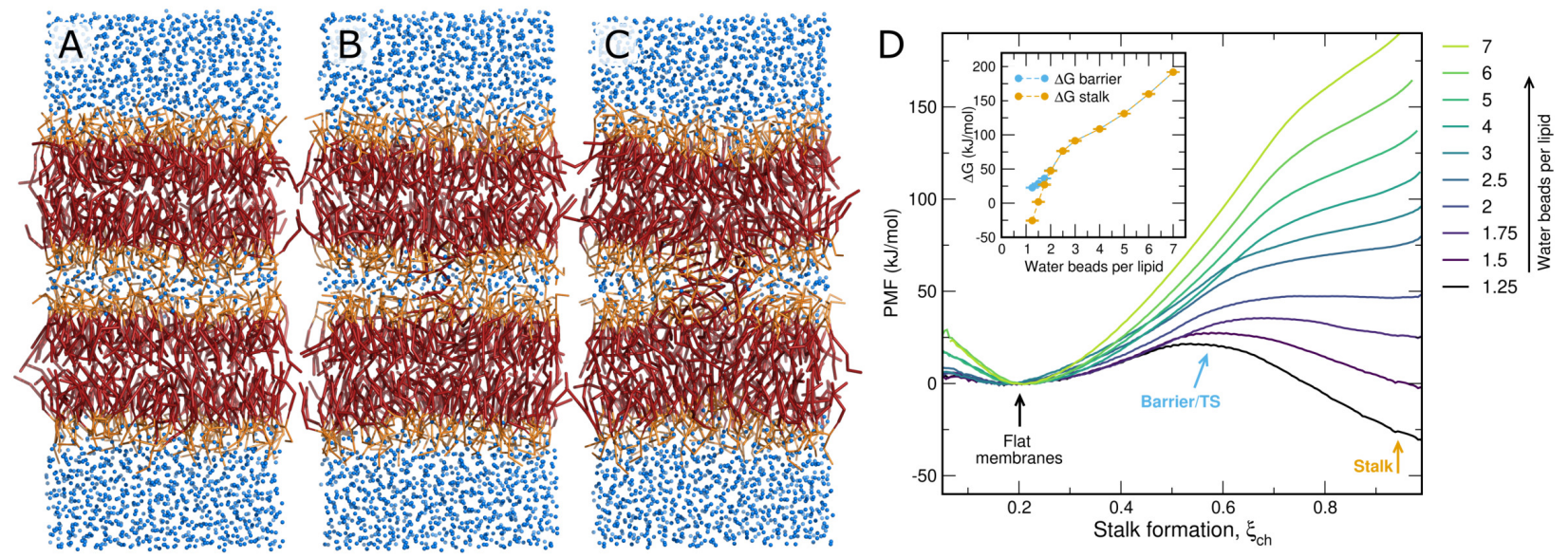

Figure 1: (A-C) Martini simulation system with membranes of pure POPC and 1.5 water beads per lipid in the proximal water compartment, showing representative frames of the (A) the flat membrane (B) the transition state (TS), and (C) the stalk. (D) PMFs of stalk formation for membranes of pure POPC, with increasing amount of water in the proximal compartment (see color code). Inset: Free energy of the stalk $\Delta G_{\text {stalk }}$ and of the stalk nucleation barrier ( $\Delta G_{\text {barrier }}$, if present) versus water beads per lipid in the proximal compartment, as taken from the PMFs.

(PMFs) along the coordinate, computed with Martini coarse-grained models, show that the inner leaflet of a common plasma membrane is far more fusogenic than the outer leaflet. To rationalize these finding, we screen the fusogenicity of lipids by varying the hydration level between the two membranes, the lipid headgroup size and charge, acyl chain length, and unsaturation levels. In addition to phosphatidylcholine (PC), PE, PS, and phosphatidylglycerol (PG) lipids, we also considered cholesterol, lysolipids, fatty acids in protonated and deprotonated forms, phosphatic acid, ceramide, diacylglycerol, and sphingomyelin. The calculations provide a comprehensive view on the role of lipid complexity during the first steps of membrane fusion. 


\section{Results}

\section{Highly efficient free energy calculations of stalk formation}

Potential of mean force (PMF) calculations may provide the free energy along reaction path in a computationally efficient manner. However, they require the choice of a good reaction coordinate (or order parameter); otherwise, undesired hysteresis effects may emerge, or energy barriers may be integrated out. We propose using a reaction coordinate for stalk formation that was originally introduced to study the formation of aqueous pores over membranes. ${ }^{28,29}$ The coordinate, named "chain coordinate" $\xi_{\mathrm{ch}}$, quantifies the degree of connectivity between two compartments. In brief, $\xi_{\text {ch }}$ is defined with the help of cylinder that spans the head group and water regions of the proximal compartment (Fig. S1). The cylinder is decomposed into $N_{s}$ slices, and $\xi_{\text {ch }}$ is defined as the fraction of slices that are filled by apolar lipid tail beads. By pulling the system along the coordinate, the slices are filled one-by-one, thereby forming a continuous apolar connection between the two fusing membranes, as required for stalk formation (Fig. 1A-C). More details are provided in the supporting material.

We used umbrella sampling along $\xi_{\mathrm{ch}}$ to compute the PMF of stalk formation in conjunction with the coarse-grained Martini lipid force field. Figure 1D presents PMFs of stalk formation across membranes of POPC lipids using different degrees of hydration in the proximal water compartment, spanning 1.25 to 7 water beads per lipid, corresponding to 5 to 28 water molecules per lipid according to the 4:1 mapping of Martini. Here, the length of

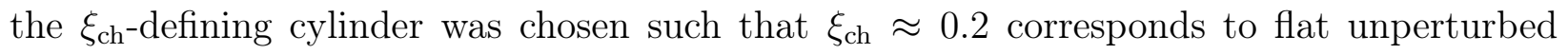
membranes, implying that $20 \%$ of the cylinder slices are filled by lipid tail beads. $\xi_{\text {ch }} \approx 1$ corresponds to a fully formed stalk. Evidently, free energy of the stalk $\Delta G_{\text {stalk relative }}$ to the flat membranes greatly depends on the degree of hydration, spanning values from $-30 \mathrm{~kJ} / \mathrm{mol}$ up to $180 \mathrm{~kJ} / \mathrm{mol}$ (Fig. 1D, inset). With fewer than 2 water beads per lipid, a barrier or transition state emerges, indicating that the stalk is metastable (long-living); with less than 1.5 water beads per lipid the stalk even forms the free energy minimum. The 
marked dependence of $\Delta G_{\text {stalk }}$ on the degree of hydration agrees with a recent simulation study, which used the string method together with the three-dimensional (3D) lipid tail density as order parameter to derive the minimum free energy path of stalk formation. ${ }^{27}$ However, with the identical simulation system, the free energies for the stalk suggested by our PMFs are significantly lower (Fig. S2), possibly because our stalk state given by $\xi_{\text {ch }} \approx 1$ includes more conformational freedom than a stalk definition via the 3D density used in Ref. 27.

The Martini model allows semi-quantitative simulations, suggesting that Martini yields trends often correctly; ${ }^{30}$ however, the exact free energy values must often be taken with care and may depend on the Martini version. To test how the stalk free energies depend on the Martini version, we re-computed the PMFs of stalk formation for POPC with the beta-3.2 release of Martini 3.0 (3.0beta) instead of Martini 2.2 (Fig. S3). Evidently, the shape of the PMFs and the dependence on hydration are well preserved among different Martini versions. However, the free energy of the stalk with Martini 3.0beta is reduced by $\sim 30 \mathrm{~kJ} \mathrm{~mol}^{-1}$ relative to Martini 2.2, suggesting that the newer Martini version is more fusogenic. Simulations with four additional lipid types confirm this trend (Fig. S4). This finding suggests that the free energies reported in this study should be interpreted in terms of trends (with hydration, degree of unsaturation etc.) and not in terms of precise free energy values.

\section{Kinetics of stalk formation agree with the PMFs}

As a critical test for the quality of the reaction coordinate and, thereby, for the validity of the PMFs, we carried out free simulations of stalk formation and stalk closure (Fig. 2). We simulated a system for which $\Delta G_{\text {stalk }} \approx 0$ according to the PMF (Fig. 2B). Among a total simulation time of $800 \mu \mathrm{s}$, we observed 8 transitions of stalk opening and 7 transitions of stalk closure, corresponding to rates of $k_{\text {stalk }}=16 \mathrm{~ms}^{-1}$ and $k_{\text {closure }}=23 \mathrm{~ms}^{-1}$, respectively. Hence, the free simulations suggest a free energy of stalk formation of $\Delta G_{\text {stalk }}=$ $-k_{B} T \ln \left(k_{\text {stalk }} / k_{\text {closure }}\right)=0.9 \mathrm{~kJ} / \mathrm{mol}$. The excellent agreement with the PMF suggests that 

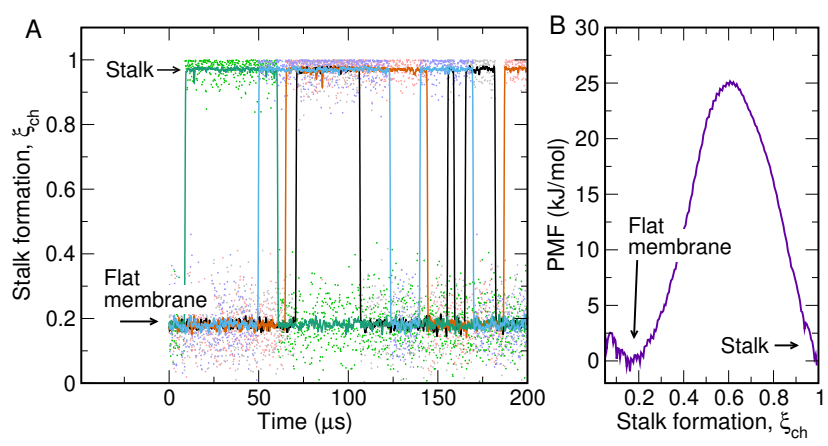

Figure 2: (A) Free simulations of stalk formation and closure. Colors indicate four independent simulations of $200 \mu \mathrm{s}$ each. $\xi_{\mathrm{ch}} \approx 0.2$ and $\xi_{\mathrm{ch}} \approx 1$ correspond to the flat membrane and the stalk, respectively. Evidently, 8 transitions of stalk formation and 7 transitions of stalk closure occurred within a total simulation time of $800 \mu \mathrm{s}$. The simulation system contained purely POPC lipids and 1.8 water beads per lipid between the proximal leaflets. Lipids were modelled with the beta-3.2 release of Martini 3.0. (B) PMF of stalk formation for the same system, revealing a free energy of stalk formation of $\Delta G_{\text {stalk }} \approx 0 \mathrm{~kJ} / \mathrm{mol}$, in excellent agreement with the free simulations.

the PMF is not affected by hysteresis problems but reports the correct $\Delta G_{\text {stalk }}$ as given by the Martini force field. Using transition state theory, the rates are expected to follow $k=\nu \mathrm{e}^{-\Delta G^{\ddagger} / k_{B} T}$, where $\nu$ is the attempt frequency, $\Delta G^{\ddagger}$ the free energy barrier in Fig. 2B, and $k_{B}$ and $T$ are the Boltzmann constant and temperature, respectively. The simulations yield $\nu \approx 0.3 \mathrm{~ns}^{-1}$, i.e., one attempt per $3 \mathrm{~ns}$, which corresponds approximately to the time scale that lipids take for traveling a lipid-lipid distance. It is interesting to note that this natural time scale for lateral lipid diffusion appears also as time scale for attempts of stalk formation.

The inner leaflet of the plasma membrane is far more fusogenic than the outer leaflet

Many critical fusion events occur with the plasma membrane. During exocytosis, transport vesicles fuse with the plasma membrane, where they first form a stalk with the inner plasma membrane leaflet. During viral infection, the envelope of certain viruses fuses with the plasma membrane, thereby forming a stalk with the outer leaflet. Like many biological membranes, the plasma membrane is asymmetric, i.e., the two leaflets exhibit different lipid 

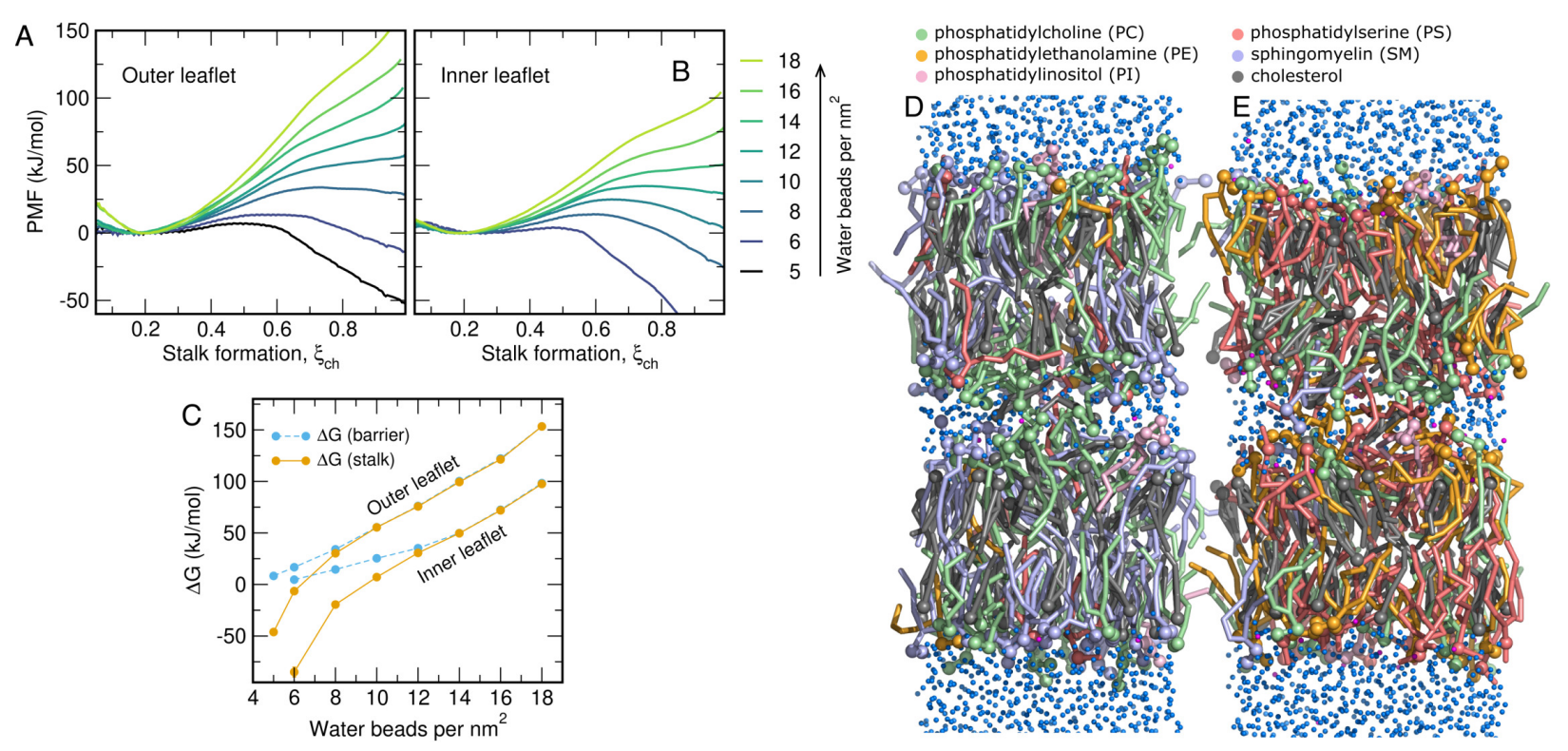

Figure 3: (A/B) PMFs of stalk formation with lipid composition taken from the plasma membrane $(\mathrm{A})$ outer leaflet or $(\mathrm{B})$ inner leaflet. $\xi_{\mathrm{ch}} \approx 0.2$ and $\xi_{\mathrm{ch}} \approx 1$ correspond to the states with planar membranes and with the stalk, respectively. PMFs were computed with increasing hydration between the proximal leaflets, between 5 and 18 water beads per $\mathrm{nm}^{2}$. (C) Free energy of the stalk (orange) and of the barrier for stalk formation (blue), taken from the PMFs in A/B. (D) Simulation frame of the open stalk in simulation with outer leaflet composition and (E) inner leaflet composition. Lipids are shown as colored sticks, headgroup beads as spheres (for color code, see legend). Water and sodium beads are shown as small blue and magenta spheres, respectively.

compositions. Further, the plasma membrane reveals a complex lipid composition, including various head group types, tail lengths, degrees of tail unsaturation, and steroid content. ${ }^{4}$

To reveal how the lipid composition of a complex biological membrane determines the free energies of stalk formation, we set up systems with symmetric membranes, but with the lipid composition mimicking either the outer or inner leaflet of a mammalian plasma membrane. ${ }^{4}$ The membranes contained phosphatidyl-choline (PC), -ethanolamine (PE), inositol (PI), -serine (PS), sphingomyelin (SM), and cholesterol, as well a various tail lengths and degrees of unsaturation, taken from a recent lipidomics study ${ }^{4}$ (Fig. 3D/E, Tables 1 and S1). The PMFs of stalk formation were again computed for various degrees of hydration in the proximal compartment quantified by the number of water beads per membrane area (Figs. 3A/B and S5). Remarkably, the free energy of stalk formation between membranes 
Table 1: Summary of lipid composition and properties in models for the outer and inner leaflet.

\begin{tabular}{lrr}
\hline & Outer & Inner \\
\hline PC & $25 \%$ & $15 \%$ \\
PE & $2 \%$ & $13 \%$ \\
SM & $25 \%$ & $2 \%$ \\
PI & $2 \%$ & $2 \%$ \\
PS & $3 \%$ & $25 \%$ \\
Cholesterol & $43 \%$ & $43 \%$ \\
\hline Saturated beads & $89 \%$ & $77 \%$ \\
Unsaturated beads & $11 \%$ & $23 \%$ \\
\hline Av. tail length (beads) & 3.9 & 4.2 \\
\hline
\end{tabular}

with the outer leaflet composition is larger by $\sim 50 \mathrm{~kJ} / \mathrm{mol}$ as compared to the membrane with the inner leaflet composition, irrespective of the degree of hydration (Figs. 3C, yellow dots). The same trend is observed for the free energy barrier for stalk formation (if present, Figs. 3C, blue dots). Hence, the inner leaflet is far more fusogenic than the outer leaflet of the plasma membrane. These different fusogenicities may reflect adaptation to the evolutionary pressure: Efficient fusion with the inner leaflet is required for exocytosis, in particular for rapid fusion of synaptic vesicles with the plasma membrane of the synapse. In contrast, an increased resistance against fusion with the outer leaflet may protect the cell against viral infection.

\section{Free energies of stalk formation are strongly influenced by tail unsaturation, tail length, and headgroup type}

Why is the inner plasma membrane leaflet more fusogenic than the outer leaflet? As listed in Tables 1 and S1, the inner leaflet model contains more PE and PS lipids, whereas the outer leaflet contains more PC and SM lipids. In addition, the lipid tails of the inner leaflet are more unsaturated as compared to the outer leaflet, as given by the fraction of beads modeling unsaturated tails (23\% versus 11\%), and the lipid tails of the inner leaflet are longer on average (4.2 versus 3.9 beads). Owing to this complexity, it is difficult to extract the key lipid properties underlying the distinct fusogenicities. 


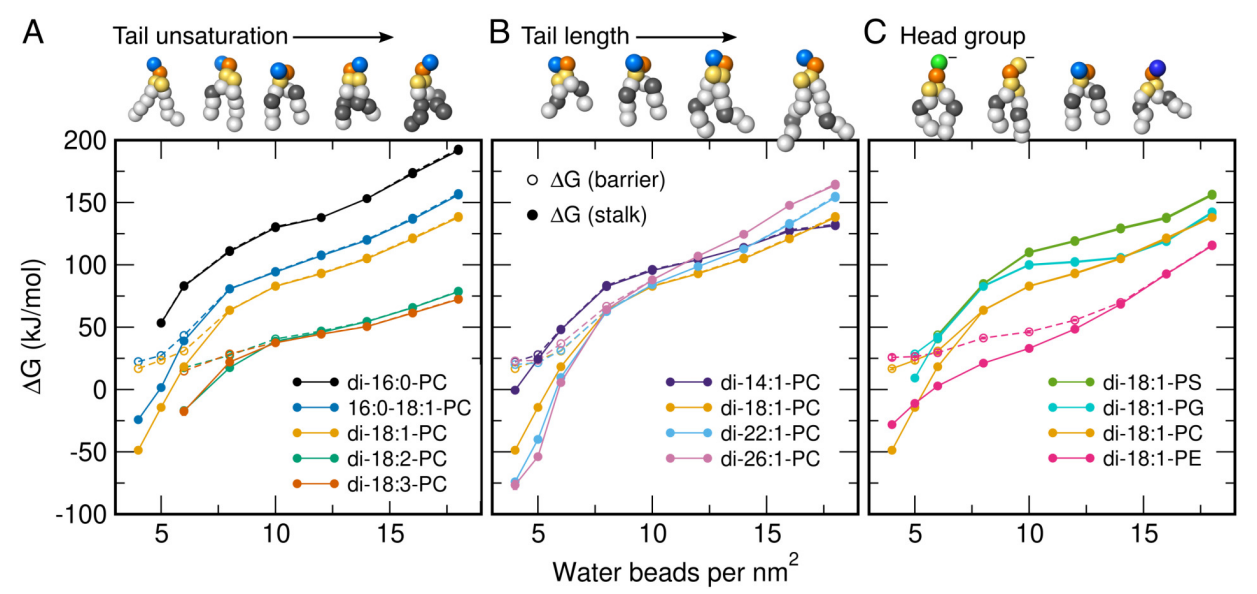

Figure 4: Free energies of stalk formation $\Delta G_{\text {stalk }}$ (solid circles) for single-lipid membranes composed of various lipid types, plotted as a function of increasing hydration between the proximal leaflets. Legends indicate the approximate atomistic correspondence of the Martini models. Open circles show the free energy barrier for stalk formation (if present). Beads of Martini lipid models are colored as follows: hydrophobic saturated (white), hydrophobic unsaturated (grey), glycerol (yellow), phosphate (orange), choline (light blue), serine (green), ethanolamine (dark blue).

To disentangle the influence of tail unsaturation, tail length, and type of head group we computed 110 additional PMFs of stalk formation. These calculations were accessible only thanks to the computational efficiency of the PMF calculations. We systematically varied either (i) the degree of unsaturation by simulating fully saturated up to poly-unsaturated tails, (ii) the tail length from 3 to 6 coarse-grained beads, thereby modeling approximately 14 to 26 carbon atoms per tail, or (iii) the type of head group between PS, PG, PC, and PE (Fig. 4A-C). The PMFs were again computed for various degrees of hydration between 4 and 18 water beads $/ \mathrm{nm}^{2}$ (Figs. S6 and S7). Molecular representations of the stalks are shown in Figs. S8, S9, and S10.

Evidently, apart from the degree of hydration, also the lipid type strongly influences the free energy of the stalk $\Delta G_{\text {stalk }}$ and the free energy barrier for stalk formation. Specifically, saturated tails as found in DPPC (di-16:0-PC) disfavor stalk formation, whereas polyunsaturated tails in lipids such as di-18:2-PC greatly favor stalk formation (Fig. 4A). In addition, increasing the tail length may stabilize the stalk at low hydration of $\leq 6$ water beads $/ \mathrm{nm}^{2}$ for which the stalk is energetically accessible (Fig. 4B). At increased hydration, however, 
for which the stalk is unstable, longer tails further destabilize the stalk. Likewise, the type of head group may influence $\Delta G_{\text {stalk }}$ : the anionic head groups of dioleoyl-PS (DOPS) and DOPG disfavor stalk formation by $\sim 25 \mathrm{~kJ} / \mathrm{mol}$ relative to the zwitterionic head group of DOPC (Fig. 4C). These findings cannot be explained merely the geometric shape of the lipids because PS headgroups are smaller than PC headgroups, whereas PG headgroups are larger than PC headgroups. Hence, the electrostatic repulsion between the anionic lipids plays a distinct role in destabilizing the stalk, possibly by effectively inducing a more positive spontaneous membrane curvature. The small zwitterionic PE headgroup facilitates stalk formation relative to the larger PC headgroup by up to $50 \mathrm{~kJ} / \mathrm{mol}$, as one may expect from the cone shape of PE lipids that may stabilize the strong negative curvature in the stalk. This trend is only inverted at very low hydration of $\leq 5$ water beads $/ \mathrm{nm}^{2}$, where stalk formation between PC membranes is more favorable than between PE membranes (Fig. 4C, yellow and magenta dots). Simulations with PE and PC membranes with alternative tails confirmed that, at most degrees of hydration, PE headgroups typically facilitate stalk formation relative to PC (Fig. S6).

Taken together, these data demonstrate that the free energy cost for stalk formation is controlled by a range of lipid properties. Namely, stalk formation is facilitated by (i) increased tail unsaturation, (ii) by longer tails at low hydration between the fusing membranes, (iii) by zwitterionic relative to anionic lipids, and (iv) by the small PE instead of the larger PC headgroup (at most degrees of hydration).

\section{$\Delta G_{\text {stalk }}$ scales linearly with lipid concentration in lipid mixtures}

Next, we investigated how the lipid type and concentration influence stalk formation in lipid mixtures. To this end, we set up addition 76 simulation systems containing POPC as reference lipid plus one type of lipid with increasing mole fraction. A constant degree of hydration with 6 water beads $/ \mathrm{nm}^{2}$ was used. In addition to the PC, PG, PS, and PE lipids considered above, and to obtain a comprehensive view on the influence of lipids on stalk free 


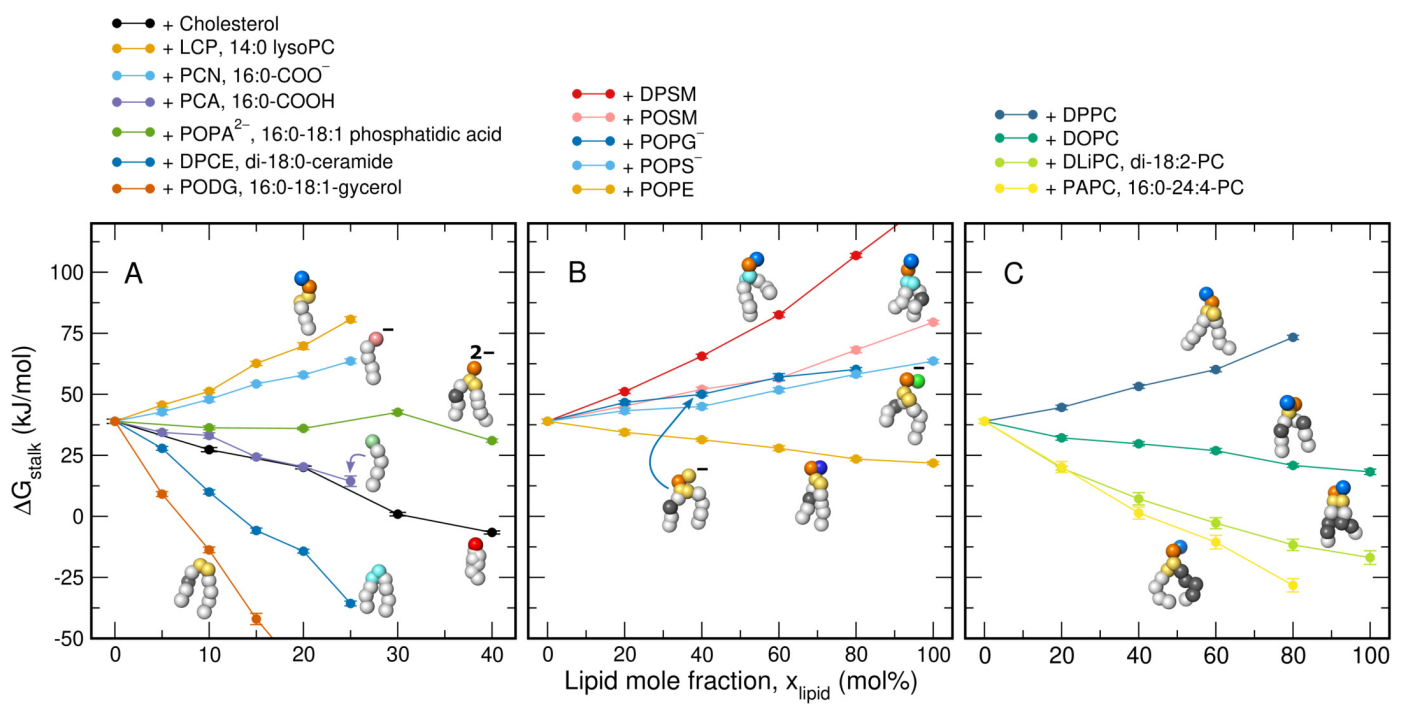

Figure 5: Free energies of stalk formation $\Delta G_{\text {stalk }}$ for binary lipid mixtures of POPC plus one additional type of lipid (for color code, see legend). 0 mol\% correspond to pure POPC. Martini beads are colored as with the scheme used for Fig. 4 and as follows: hydroxyl (red), carboxylate (pale red), carboxyl (pale green), sphingosine (cyan).

energies, we considered a wide range of additional lipids: cholesterol, lyso lipids, fatty acids, phosphatidic acid (PA), ceramide, diacylglycerol, and sphingomyelin (SM).

Figure 5 presents $\Delta G_{\text {stalk }}$ as function of mole fraction $x_{\text {lipid }}$ for various lipid types, where $x_{\text {lipid }}=0 \%$ corresponds to pure POPC. Typical PMFs are presented in Fig. S11. Evidently, mixing a second type of lipid into a POPC membrane may greatly stabilize or destabilize the stalk, while $\Delta G_{\text {stalk }}$ depends nearly linearly on the mole fraction of the second lipid. Here, the slope of the $\Delta G_{\text {stalk }}-x_{\text {lipid }}$ curves quantify the sensitivity of stalk formation upon the addition of a second lipid.

As expected, the geometric shape of the lipid, that is whether the lipid is cone- or inverted cone-shaped, affects $\Delta G_{\text {stalk }}$. Only small amounts of ceramide and diacylglycerol greatly stabilize stalks, compatible with their highly negative intrinsic curvature (Fig. 5A, dark blue, dark orange). On the contrary, single-chained lysoPC greatly destabilizes the stalk, compatible with its large positive curvature (Fig. 5A, orange). Single-chained fatty acids stabilize the stalk in the protonated form 16:0-COOH, however they destabilize the stalk in the deprotonated form 16:0-COO- (Fig. 5A, light blue, purple). Owing to the strongly 
increased $\mathrm{pK}_{\mathrm{a}}$ of fatty acids in lipid membranes relative to bulk water, the protonated, stalk-stabilizing form is predominantly present in biological membranes. ${ }^{31}$

In agreement with the findings for single-component membranes presented above, $\Delta G_{\text {stalk }}$ is modulated by replacing PC head groups of POPC with other head groups while maintaining the palmitoyl-oleoyl tails. Whereas replacing $\mathrm{PC}$ with $\mathrm{PA}$ has only a small effect on $\Delta G_{\text {stalk, }}$ replacing $\mathrm{PC}$ with $\mathrm{PE}$ favors stalk formation (Fig. 5A, green, Fig. 5B, orange). In contrast, replacing PC with the anionic PS or PG head groups disfavors stalk formation (Fig. 5B, light and dark blue). Likewise, sphingomyelin disfavors stalk formation, in particular the fully saturated DPSM. These findings are rationalized by the tendency of sphingomyelin of increasing the order of the aliphatic tails, thereby disfavoring the formation of a defect such as a stalk. In addition, the $\Delta G_{\text {stalk }}$ values from lipid mixtures confirm that the addition of (poly-)unsaturated lipid tails facilitate stalk formation, whereas the addition of saturated tails hinder stalk formation (Fig. 5C). Finally, the addition of cholesterol strongly stabilizes the stalk, by up to $45 \mathrm{~kJ} / \mathrm{mol}$ at $40 \mathrm{~mol} \%$ cholesterol, in qualitative agreement with results from X-ray diffraction. ${ }^{32}$

\section{Stalk stabilization correlates with specific lipid enrichment in the stalk, except for cholesterol}

To shed light on the molecular mechanism by which different lipids influence $\Delta G_{\text {stalk }}$, we computed the lipid densities in simulations with a fully formed stalk (Figs 6A/B and S12S15). The overall shape of the stalk is largely independent of the lipid composition, except that energetically unstable stalks are slightly thinner as compared to stalks that form the free energy minimum. However, the lipid composition strongly influences the spatial distribution of the individual lipid types. For instance, the stalk-stabilizing DLiPC is enriched at the stalk, whereas stalk-destabilizing DPSM is depleted at the stalk (Figs 6A/B, left column). To test whether these trends hold for other lipids, we quantified the enrichment of a lipid in the stalk as $\rho_{\text {stalk }} / \rho_{\text {mem }}-1$, where $\rho_{\text {stalk }}$ and $\rho_{\text {mem }}$ denote the average lipid mass densities at the 

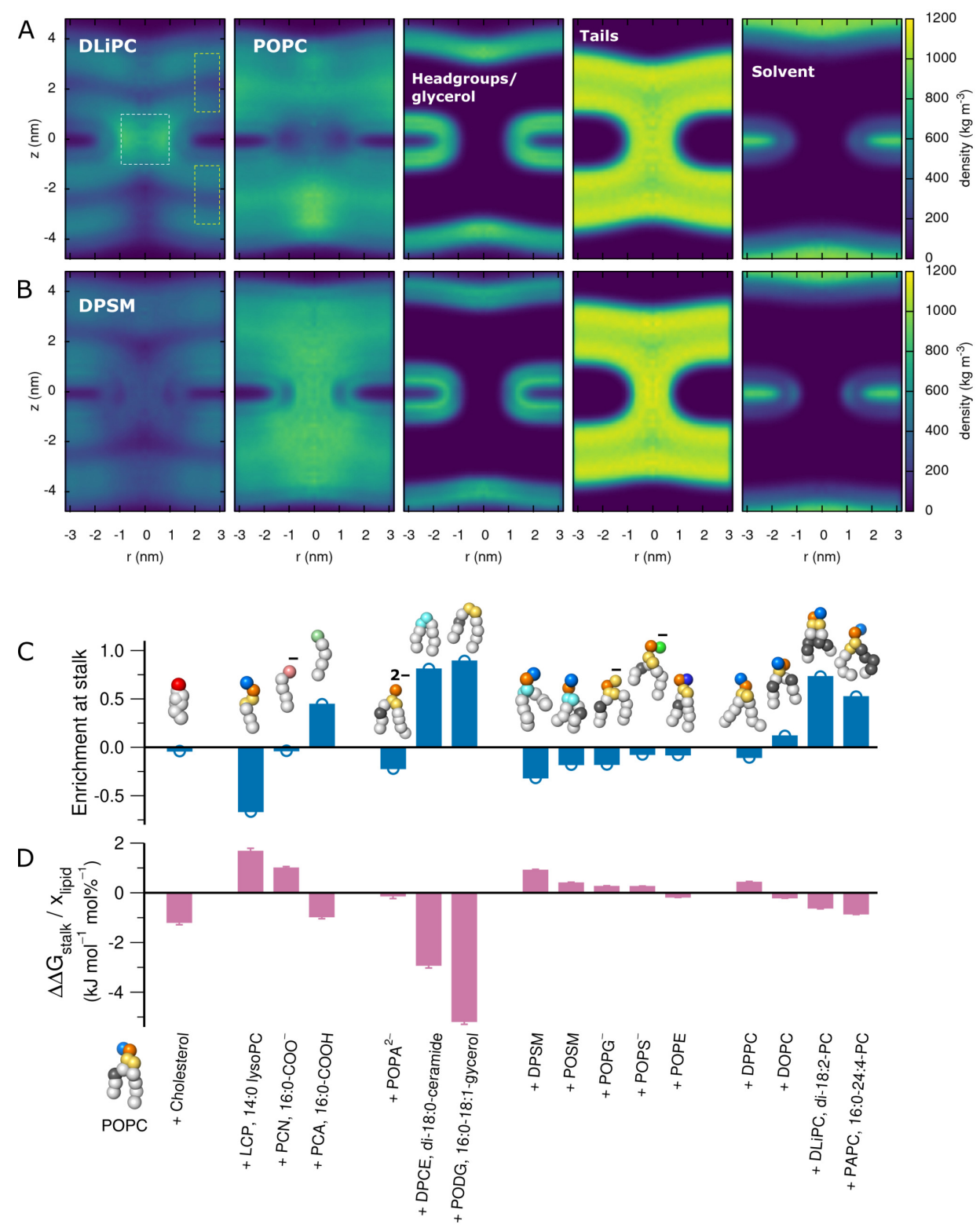

Figure 6: (A) Mass densities of (from left to right) DLiPC, POPC, headgroups plus glycerol beads, lipid tails, and solvent in the fully formed stalk in a system with a POPC:DLiPC ratio of 60:40. (B) Same as in panel (A), but for the DPSM:POPC 60:40 system. (C) Enrichment of lipids inside the stalk relative to the flat membrane: $\rho_{\text {stalk }} / \rho_{\text {mem }}-1$, where $\rho_{\text {stalk }}$ and $\rho_{\text {mem }}$ are the average densities in the white and yellow boxes in panel (A), respectively. Beads of Martini lipid models are colored as follows: hydrophobic saturated (white), hydrophobic unsaturated (grey), hydroxyl (red), glycerol (yellow), phosphate (orange), choline (light blue), carboxylate (pale red), carboxyl (pale green), sphingosine (cyan), serine (green), ethanolamine (dark blue). (D) Change of stalk free energy $\Delta \Delta G_{\text {stalk }}$ per lipid concentration $c_{\text {lipid }}$ upon addition of lipid to a POPC membrane. 
stalk and in a region of the flat membrane, respectively (Figs 6A, white and yellow boxes). Hence, a positive and negative values indicate lipid enrichment or depletion at the stalk, respectively. In addition, we quantified the influence of specific lipids on stalk formation as the slope of a linear least-square fit to the data in Fig. 5 , denoted $\Delta \Delta G_{\text {stalk }} / x_{\text {lipid }}$.

Evidently, among nearly all lipids, lipid enrichment in the stalk anti-correlates with the $\Delta \Delta G_{\text {stalk }} / x_{\text {lipid }}$. Hence, lipids that either favor negative curvature, increased tail disorder, or both, may accumulate in the stalk, thereby stabilizing the stalk structure. However, there are notable outliers: cholesterol stabilizes the stalk without being enriched in the stalk. Instead, in line with previous reports, ${ }^{33}$ cholesterol is even slightly depleted at the stalk, possibly because the planar cholesterol molecule disfavors the increased tail disorder in the stalk. These findings suggest that cholesterol does not favor stalk formation owing to an intrinsic negative curvature of cholesterol, but more likely owing to its influence on the dehydration repulsion between the membranes.

Another outlier is diacyl-glycerol PODG, the by far most stalk-stabilizing lipid considered in this study. The lipid densities show that PODG accumulated not only inside the stalk, but mostly on top and below the stalk within the membrane core (Fig. S13). The accumulation within the membrane core is possible because the headgroup of diacyl-glycerol is only weakly polar. We hypothesize that similar effects play a role during biogenesis of lipid droplets, which contain large amounts of triacylglycerol and which are released from the ER membrane via a stalk-like intermediate. ${ }^{34}$

\section{Discussion}

The marked difference between the outer and inner leaflet prompted us to screen free energies of stalk formation for a wide range of single-lipid membranes as well as for membranes of binary lipid mixtures. The results provided a comprehensive and quantitative fusogenicity map of lipids, as summarized in Fig. 6D. The data suggest that free energies of stalk 
formation are not determined by a single lipid property, but instead by a combination of molecular mechanisms: (i) Stalk formation is facilitated by increased tail disorder, as present in polyunsaturated lipids, whereas increased tail order hinders stalk formation, as common for sphingomyelin. (ii) As expected from previous studies, ${ }^{35}$ lipids with negative intrinsic curvature promote stalk formation as they may stabilize the large negative curvature of the stalk. Such mechanism applies to protonated fatty acids, to PE lipids and ceramide owing to their small head groups, as well as to polyunsaturated lipids owing to the increased tail volume. A counter example is given by lysoPC that hinders stalk formation owing to its positive intrinsic curvature. ${ }^{35}$ (iii) Likewise, we find that anionic headgroups counteract stalk formation. The simulations demonstrate that anionic headgroups counteract stalk formation, as implied by increased $\Delta G_{\text {stalk }}$ for PS or deprotonated fatty acids despite of their small head groups. In line with this observation, POPA hardly affects $\Delta G_{\text {stalk }}$ despite the very small headgroup. These trends are likewise rationalized by the electrostatic repulsion between headgroups, which effectively disfavor negative curvature. ${ }^{36}$ However, since polar interactions are less well represented by the Martini coarse-grained force field as compared to apolar interactions, it will be interesting to test these predictions by experiments and by atomistic simulations. (iv) Cholesterol facilitates stalk formation. However, since cholesterol is not enriched in the stalk, the stalk-stabilizing effect of cholesterol can not rely on a putative stabilization of the negative curvature in the stalk, as suggested previously. ${ }^{37}$ The effect of cholesterol is also surprising considering that cholesterol increases the tail order of membranes, similar to the sphingomyelin that, in contrast to cholesterol, increases $\Delta G_{\text {stalk }}$. Therefore, we hypothesize that cholesterol may promote stalk formation by reducing the hydration repulsion, ${ }^{38}$ thereby facilitating the formation of the first contacts between the fusing leaflets.

By simulating stalk formation between membranes with complex lipid composition, we found that the inner leaflet of a typical mammalian plasma membrane ${ }^{4}$ is more fusogenic than the outer leaflet by $\sim 50 \mathrm{~kJ} / \mathrm{mol}$. These properties may be an adaption to evolutionary 
pressure: a fusogenic inner leaflet facilitates exocytosis, thereby increasing the fusion rates and reducing the required energy consumption by fusion proteins. A less fusogenic outer leaflet might hinder infection by enveloped viruses that fuse with the plasma membrane. The fusogenicity of lipids in Fig. 6D provides the molecular rationale for the distinct fusogenicities of the inner and outer leaflets. Namely, the increased fusogenicity of the inner leaflet is mainly caused by the increased number of polyunsaturated tails, mainly present as PS and PE lipids, and, to a lower degree, by the large PE content (cf. Table 1). The outer leaflet counteracts fusion mainly owing to the large sphingomyelin content, fewer polyunsaturated tails, and increased PC content relative to PE.

These conclusions were obtained with novel reaction coordinate for stalk formation, which allowed computationally highly efficient free energy calculations of stalk formation along thermodynamically reversible pathways. The PMFs converge rapidly, are not affected by hysteresis problems, and they yield the true free energy difference between the lamellar and the stalk state for the given force field, as confirmed by free simulations. One PMF required less than 7 hours on an inexpensive server equipped with a 6-core CPU and a consumer graphics card, hence allowing for high-throughput calculations. Our PMF calculations complement computationally more elaborate methods such as the string method, which is capable of finding the minimum free energy path of stalk formation without the need of identifying a good reaction coordinate. ${ }^{27}$ The computational efficiency further relied on the use of the Martini coarse-grained force field that achieves a speedup in sampling by a factor of $\sim 1000$ relative to atomistic simulations due to fewer particles, longer integration time step, and accelerated lipid diffusion. However, with the new reaction coordinate, free energy calculations of stalk formation with atomistic MD simulations are now within reach. This will allow us to test whether coarse-grained simulation primarily provide trends or whether they also provide quantitatively precise predictions of free energies during fusion.

While this study focused on stalk formation, it is evident that other stages of the fusion process are influenced by the lipid composition as well. Stalk widening as well as opening 
and expansion of the fusion pore involve highly curved membranes and are therefore controlled by lipid curvature. ${ }^{10,39}$ Hence, future studies should quantify the role of complex lipid compositions on such later stages of fusion, in addition to lipid effects on the dehydration repulsion, which may add a free energy offset to the free energies of stalk formation computed here. ${ }^{27}$ Further, it will be interesting to test whether lipid-protein interactions guide a local enrichment of fusogenic lipids and, thereby, further facilitate fusion.

\section{Conclusions}

Using a newly developed method for computing free energies of stalk formation $\Delta G_{\text {stalk }}$, we presented a comprehensive and quantitative fusogenicity map of lipids. The simulations showed that the lipid composition of membranes may modulate $\Delta G_{\text {stalk }}$ by $100 \mathrm{~kJ} / \mathrm{mol}$ or even more. A range of lipid properties facilitate stalk formation, including increased tail unsaturation, longer tails, smaller head groups, and cholesterol content. In contrast, stalk formation is hindered by anionic lipids and by lipids that increase the tail order such as sphingomyelin. We found that the lipid composition of the inner leaflet of a mammalian plasma membrane greatly favors stalk formation relative to the outer leaflet. The distinct fusogenicities of the two leaflets is likely an adaptation to physiological requirements and is mainly rationalized by the different content of polyunsaturated lipids, sphingomyelin, and PE lipids.

\section{Methods Summary}

MD simulations were carried out with Gromacs, version 2020.3, ${ }^{40}$ and with an in-house modification of Gromacs 2018.8 that implements the harmonic restraint along the reaction coordinate $\xi_{\mathrm{ch}}$, originally introduced to study pore formation over membranes. ${ }^{28,29}$ PMFs were computed with umbrella sampling using 19 umbrella windows and simulating each window for $200 \mathrm{~ns}$. Interactions were described with the Martini coarse-grained force field 
version 2.2 if not stated otherwise. ${ }^{41}$ Details on the reaction coordinate, simulation setup, parameters, and analysis are provided in the supporting material.

\section{Acknowledgement}

We thank Yuliya Smirnova and Jelger Risselada for insightful discussions, for sharing the PChd200 and PCh220 simulation systems, and for critical comments on the manuscript. This study was supported by the Deutsche Forschungsgemeinschaft via SFB 1027/B7.

\section{References}

(1) van Meer, G.; Voelker, D. R.; Feigenson, G. W. Membrane lipids: where they are and how they behave. Nat Rev Mol Cell Biol 2008, 9, 112-124.

(2) Shevchenko, A.; Simons, K. Lipidomics: coming to grips with lipid diversity. Nat Rev Mol Cell Biol 2010, 11, 593-598.

(3) Op Den Kap, J. A. F. Lipid asymmetry in membranes. Ann. Rev. Biochem. 1979, 48, 47-71.

(4) Lorent, J. H.; Levental, K. R.; Ganesan, L.; Rivera-Longsworth, G.; Sezgin, E.; Doktorova, M.; Lyman, E.; Levental, I. Plasma membranes are asymmetric in lipid unsaturation, packing and protein shape. Nat Chem Biol 2020, 16, 644-652.

(5) Jahn, R.; Lang, T.; Südhof, T. C. Membrane fusion. Cell 2003, 112, 519-533.

(6) Wickner, W.; Schekman, R. Membrane fusion. Nat Struct Mol Biol 2008, 15, 658-664.

(7) Harrison, S. C. Viral membrane fusion. Nat Struct Mol Biol 2008, 15, 690-698.

(8) Kielian, M.; Rey, F. A. Virus membrane-fusion proteins: more than one way to make a hairpin. Nat Rev Microbiol 2006, 4, 67-76. 
(9) Markin, V. S.; Kozlov, M. M.; Borovjagin, V. L. On the theory of membrane fusion. The stalk mechanism. Gen Physiol Biophys 1984, 3, 361-377.

(10) Chernomordik, L. V.; Kozlov, M. M. Mechanics of membrane fusion. Nat Struct Mol Biol 2008, 15, 675-683.

(11) Martens, S.; McMahon, H. T. Mechanisms of membrane fusion: disparate players and common principles. Nat Rev Mol Cell Biol 2008, 9, 543-556.

(12) Burger, K. N. Greasing Membrane Fusion and Fission Machineries. Traffic 2000, 1, 605-613.

(13) Jahn, R.; Südhof, T. C. Membrane Fusion and Exocytosis. Annu. Rev. Biochem. 1999, $68,863-911$.

(14) Antonny, B.; Vanni, S.; Shindou, H.; Ferreira, T. From zero to six double bonds: phospholipid unsaturation and organelle function. Trends in Cell Biology 2015, 25, 427-436.

(15) Kozlovsky, Y.; Kozlov, M. M. Stalk Model of Membrane Fusion: Solution of Energy Crisis. Biophysical Journal 2002, 82, 882-895.

(16) Noguchi, H.; Takasu, M. Fusion pathways of vesicles: A Brownian dynamics simulation. The Journal of Chemical Physics 2001, 115, 9547-9551.

(17) Müller, M.; Katsov, K.; Schick, M. New mechanism of membrane fusion. The Journal of Chemical Physics 2002, 116, 2342-2345.

(18) Katsov, K.; Müller, M.; Schick, M. Field Theoretic Study of Bilayer Membrane Fusion. I. Hemifusion Mechanism. Biophysical Journal 2004, 87, 3277-3290.

(19) Smirnova, Y. G.; Marrink, S.-J.; Lipowsky, R.; Knecht, V. Solvent-Exposed Tails as Prestalk Transition States for Membrane Fusion at Low Hydration. J. Am. Chem. Soc. 2010, 132, 6710-6718. 
(20) Risselada, H. J.; Kutzner, C.; Grubmüller, H. Caught in the Act: Visualization of SNARE-Mediated Fusion Events in Molecular Detail. ChemBioChem 2011, 12, 10491055 .

(21) Kawamoto, S.; Shinoda, W. Free energy analysis along the stalk mechanism of membrane fusion. Soft Matter 2014, 10, 3048.

(22) Markvoort, A. J.; Marrink, S. J. Current Topics in Membranes; Elsevier, 2011; Vol. 68; pp 259-294.

(23) Schick, M. Membrane Fusion: the Emergence of a New Paradigm. J Stat Phys 2011, 142, 1317-1323.

(24) Risselada, H. J.; Grubmüller, H. How SNARE molecules mediate membrane fusion: recent insights from molecular simulations. Curr. Opin. Struct. Biol. 2012, 22, 187196.

(25) Marrink, S. J.; Mark, A. E. The Mechanism of Vesicle Fusion as Revealed by Molecular Dynamics Simulations. J. Am. Chem. Soc. 2003, 125, 11144-11145.

(26) Kasson, P. M.; Pande, V. S. Control of Membrane Fusion Mechanism by Lipid Composition: Predictions from Ensemble Molecular Dynamics. PLoS Comput Biol 2007, 3, e220.

(27) Smirnova, Y. G.; Risselada, H. J.; Müller, M. Thermodynamically reversible paths of the first fusion intermediate reveal an important role for membrane anchors of fusion proteins. Proc Natl Acad Sci USA 2019, 116, 2571-2576.

(28) Hub, J. S.; Awasthi, N. Probing a continuous polar defect: A reaction coordinate for pore formation in lipid membranes. J. Chem. Theory Comput. 2017, 13, 2352-2366.

(29) Ting, C. L.; Awasthi, N.; Müller, M.; Hub, J. S. Metastable Prepores in Tension-Free Lipid Bilayers. Phys. Rev. Lett. 2018, 120. 
(30) Marrink, S. J.; Tieleman, D. P. Perspective on the Martini model. Chem. Soc. Rev. 2013, 42, 6801.

(31) Kurniawan, J.; Suga, K.; Kuhl, T. L. Interaction forces and membrane charge tunability: Oleic acid containing membranes in different pH conditions. Biochimica et Biophysica Acta (BBA) - Biomembranes 2017, 1859, 211-217.

(32) Aeffner, S.; Reusch, T.; Weinhausen, B.; Salditt, T. Energetics of stalk intermediates in membrane fusion are controlled by lipid composition. Proceedings of the National Academy of Sciences 2012, 109, E1609-E1618.

(33) Risselada, H. J.; Bubnis, G.; Grubmuller, H. Expansion of the fusion stalk and its implication for biological membrane fusion. Proceedings of the National Academy of Sciences 2014, 111, 11043-11048.

(34) Dhiman, R.; Caesar, S.; Thiam, A. R.; Schrul, B. Mechanisms of protein targeting to lipid droplets: A unified cell biological and biophysical perspective. Seminars in Cell \&6 Developmental Biology 2020, 108, 4-13.

(35) Chernomordik, L. V.; Kozlov, M. M. Membrane Hemifusion: Crossing a Chasm in Two Leaps. Cell 2005, 123, 375-382.

(36) Fuller, N.; Benatti, C. R.; Peter Rand, R. Curvature and Bending Constants for Phosphatidylserine-Containing Membranes. Biophysical Journal 2003, 85, 1667-1674.

(37) Yang, S.-T.; Kreutzberger, A. J.; Lee, J.; Kiessling, V.; Tamm, L. K. The role of cholesterol in membrane fusion. Chemistry and Physics of Lipids 2016, 199, 136-143.

(38) McIntosh, T. J.; Magid, A. D.; Simon, S. A. Cholesterol modifies the short-range repulsive interactions between phosphatidylcholine membranes. Biochemistry 1989, 28, $17-25$. 
(39) Dhara, M.; Mantero Martinez, M.; Makke, M.; Schwarz, Y.; Mohrmann, R.; Bruns, D. Synergistic actions of v-SNARE transmembrane domains and membrane-curvature modifying lipids in neurotransmitter release. eLife 2020, 9, e55152.

(40) Abraham, M. J.; Murtola, T.; Schulz, R.; Páll, S.; Smith, J. C.; Hess, B.; Lindahl, E. GROMACS: High performance molecular simulations through multi-level parallelism from laptops to supercomputers. SoftwareX 2015, 1, 19-25.

(41) Marrink, S. J.; Risselada, H. J.; Yefimov, S.; Tieleman, D. P.; de Vries, A. H. The MARTINI force field: coarse grained model for biomolecular simulations. J. Phys. Chem. B 2007, 111, 7812-7824. 